\title{
Study on ANT-based Wireless Network for Wearable Medical Monitoring
}

\author{
Niu Fu Rong ${ }^{1}$ and Yan Yi Xin ${ }^{2 *}$ \\ Harbin University of Science and Technology, Harbin, 150080, P. R. China \\ ${ }^{1} 1353788227 @ q q . c o m,{ }^{2 *}$ shiyu_wang@126.com
}

\begin{abstract}
Such technical features of ANT as protocol stack structure, data frame format and channel configuration are first introduced in the paper, and then targeted at the groups that need real-time medical monitoring, an ANT protocol-based wireless network medical monitoring system is established to realize real-time acquisition of corresponding physiological data. This system adopts the chip NRF24AP2 compatible with ANT protocol as the core component of wireless network nodes of the medical monitoring system to conduct design of software and hardware. It is shown through power consumption analysis and communication reliability testing of the wireless network that this network features low power consumption under the premise of operating reliability, applicable to various applications giving priority to low power consumption.
\end{abstract}

Keywords: ANT; NFR24AP2; wireless network; wearable

\section{Introduction}

With the aggravation of aging problems in the world and people's concern about health, medical health care monitoring has become the focus of public discussion. At the same time, in order to reduce the cost of medical monitoring, improve monitoring quality and provide convenience for special groups that need to receive long-term medical monitoring, such as patients suffering from chronic diseases, post-surgery patients and pregnant women, a new kind of monitoring pattern is needed. Wearable medical monitoring instruments feature small size, light weight, low cost, carrying convenience, etc. Such information as various kinds of physiological signals and medical data about wearers can be transmitted to the designated terminals via wireless network to obtain preliminary diagnostic schemes and provide patients with some reminders, thereby realizing the medical information sharing and exchanges between patients and hospitals and between urban hospital groups. Therefore, the development of wearable medical monitoring technology can make up for the shortage of medical resources (such as the number of hospitals, medical care personnel) in the same period [1].

ANT protocol is a new wireless network communication protocol, short- distance nonstandard RF Protocol [2], and 2.4Hz ultra low-power wireless network standard launched by such companies as Nordic and Dynastream in 2005, able to realize the vast majority of application scenarios of ZigBee [3], featuring low cost, low power consumption, easy application, flexibility, reliability, shorter development cycle and no need to pay, with great competitive performance advantage in terms of power consumption, system cost and development cycle. 


\section{ANT Protocol}

\subsection{Introduction to ANT}

ANT protocol supports multi-frequency and high-density networks, with an air data rate of $1 \mathrm{Mbit} / \mathrm{s}$ and a transmission distance of $10 \square 30 \mathrm{~m}$. ANT has low cost and adopts adaptive synchronous channel, point to point and point to multipoint transmission modes, with a network data rate of up to $20 \mathrm{kbit} / \mathrm{s}$ and strong anti-interference ability. ANT adopts a peer-to-peer network model, with each node having the same circuit structure and network functions, undertaking difference tasks in the network based on different configurations. ANT supports point-to-point, star-form, tree-form and other network topology structures, with the characteristics of reliability and flexibility. TDMA mode is used for ANT wireless signals, supporting unidirectional or bi-directional channels, with data transmission mode either a fixed mode or Ad-hoc mode.

\subsection{Protocol Stack of ANT}

ANT network configuration and control are very convenient, with ANT protocol stack as shown in Figure 1. Manufacturers encapsulate ANT protocol stacks in the chips in advance, involving the processing of the physical layer, data link layer, network layer and transmission layer and lower-level network security mechanism. The application layer software designed by developers merely needs simple configuration ANT chips, thus completing operations like networking and communication, with no such skills as adept programming required [4].

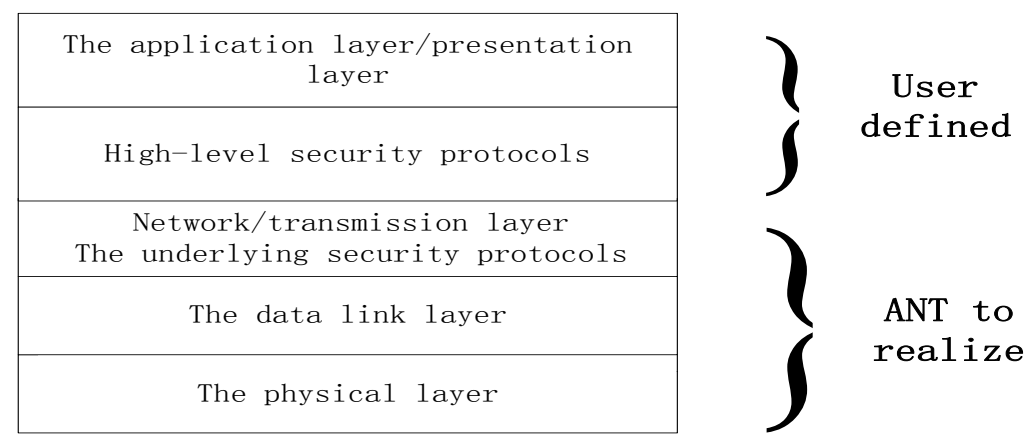

Figure 1. OSI Layer Model of ANT

\subsection{Interface}

A synchronous or asynchronous serial mode and common I/O port can be adopted for the hardware communication interface between the external processor and the ANT chip, and as for software interface, the processer can communicate with the ANT chip in an information-driven mode: the processor sends commands in a required format to the ANT chip to realize network configuration and data transmission, and in turn the ANT chip can also send data frames to the processor in the required format when it receives RF data sent by other nodes. As for ANT serial data frames, bytes are transmitted in a low preamble mode, beginning with synchronization code and ending with verification code, with basic format as shown in Table1. 
Table1. ANT Serial Data Frame Format

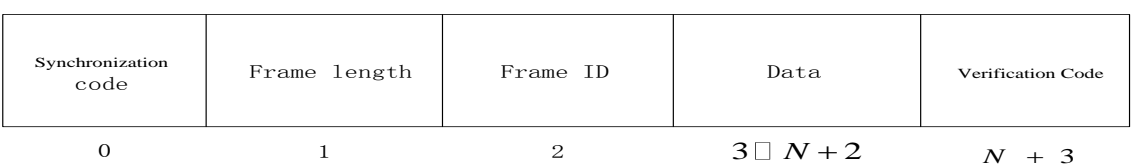

The meanings of data frame components are as follows:

Synchronization Code (1 byte): transmission direction of serial data frames. 0xA5 indicates the processor transmits data to the ANT chip, and 0xA4 indicates the ANT chip transmits data to the processor.

Frame Length ( 1 byte): number of bytes of user data, not more than 9 bytes.

Frame ID (1 byte): data frame type, valid value: $1 \square 255$.

Data ( $\mathrm{N}$ bytes): user-defined communication data.

Verification Code (1 byte): equal to the XOR value of all previous data.

\subsection{Channel Configuration of ANT Protocol}

\subsubsection{Channel and Node}

ANT realizes network management and communication control based on channel and any two of ANT nodes are connected through special channels, which serve as medium for information transmission. In ANT protocol, each channel has at least 1 master node and 1slave node (the master-slave node relationship is determined by the main flow direction of data between them, with the main output nodes of data being master nodes and main input nodes of data as slave nodes) and the adoption of the TDMA mechanism for ANT protocol allows a certain node to serve as a master node of on channel and a slave node of another channel at the same time.

ANT network has two kinds of channels, independent channels and shared channels: an independent channel includes 1 master node and 1 slave node, and a share channel consists of 1 master node and several slave nodes. A certain node in ANT can belong to several network channels at the same time and play different network roles in different channels. Through this kind of networking mode, ANT nodes can serve not only as the active sender of data packets but also as the passive receiver of data packet or become the routing nodes of relay data packets. ANT nodes can join or leave the network at any time, with no effect on network topology or data transmission.

\subsubsection{Channel Configuration}

Before ANT nodes realize communication, configuration of channel parameters must be conducted, with the channel parameters of ANT network including:

(1) Network ID: ANT protocol network types: private, under management and open. The network types of ANT channels are set by setting network ID and network key.

(2) RF Frequency: the center frequency of wireless communication of channels is specified. ANT supports a total of 125 working frequencies. In the work mode involving no use of frequency hopping, when the working frequency of a channel is set, it will continuously work at the fixed working frequency, with the RF frequency setting formula as follows:

$$
\text { the } R F \text { frequency settings }=\frac{\text { Working frequency channel }(\mathrm{MHZ})-2400(\mathrm{MHZ})}{1(\mathrm{MHZ})}
$$

(3) Channel ID: it is the most complex and most flexible one amount channel parameters, including equipment ID, equipment type and transmission type. 
(4) Channel Type: ANT protocol network channels include single sending channels, single receiving channels, bi-directional channels and shared bi-directional channels, with the basic transmission direction of data in the channel specified.

(5) Channel Cycle: the cycle for the host in the channel to send data at fixed time. The data transmission frequency scope of ANT channels is $0.5 \sim 200 \mathrm{HZ}$. The calculation formula of channel cycle is as follows:

$$
\text { Cycle Settings }=\frac{32768}{\text { Data transmission frequency }(\mathrm{HZ})}
$$

\section{Overall Structure and Working Principle of ANT Sensor Network}

The wearable medical monitoring system based on ANT protocol mainly consists of sensor nodes, central node, routing node and medical center server [5]. Figure 2 is a general block diagram of wireless network, where the sensor nodes are mainly used to realize real-time acquisition of all parameters and send data to the central node. The central node bring together various data sent back from the sensor nodes in an ANT star network and then sends the gathered data to the routing node. The routing node sends all data from the central node to the data monitoring and control terminal to facilitate the server terminals' real-time monitoring of each monitored terminal and receiving of commands sent from the service center. Sensor nodes and central nodes are generally worn on the user's body and the routing nodes are placed in family communities and hospital communities. Each node has an ANT protocol-based wireless transceiver module, realizing wireless network-based communication by sending and receiving wireless data.

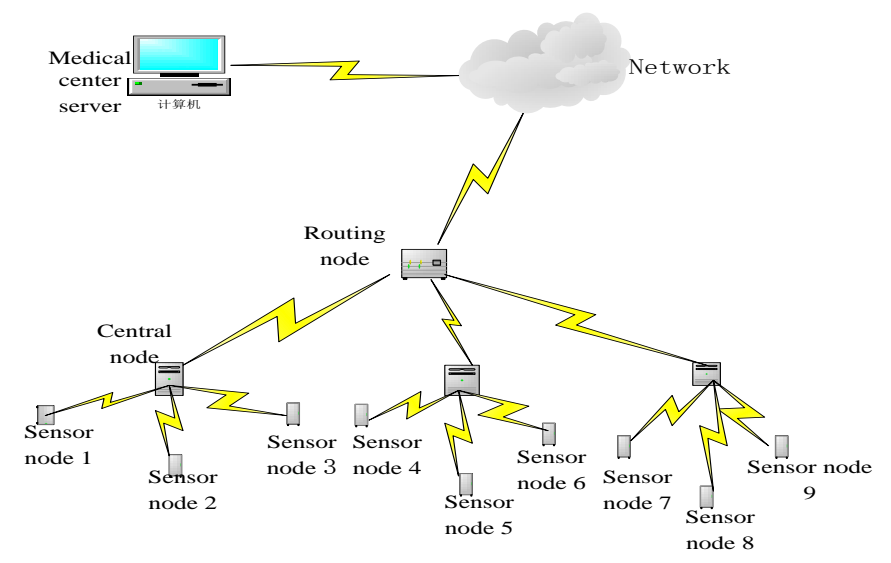

Figure 2. General Block Diagram of Wireless Network

The hardware part of the wearable medical monitoring system based on ANT Protocol mainly consists of three parts, sensor nodes, central nodes and routing nodes [6], of which:

(1) Sensor Node [7]: a sensor node consists of a vital sign sensor [8], microcontroller MSP430F5418A [9], ANT wireless communication module nRF24AP2-1CH and CR2032 lithium ion button cell battery [10]. Figure 3 is a structure diagram of a sensor node. MSP430F5418A is a 16-bit ultra-low power MCU of TI company, with diverse external device functions, integrating such communication interfaces as UART, SPI and $I^{2} C$, facilitating interconnection with external systems. nRF24AP2-1CH is a single channel ANT wireless networking chip used for data sending and receiving before nodes. An asynchronous serial mode is used for the communication between the micro-controller 
and the $\mathrm{d}$ wireless communication module, with working voltage being $3 \mathrm{~V}$, supplied by CR2032 lithium ion button battery.

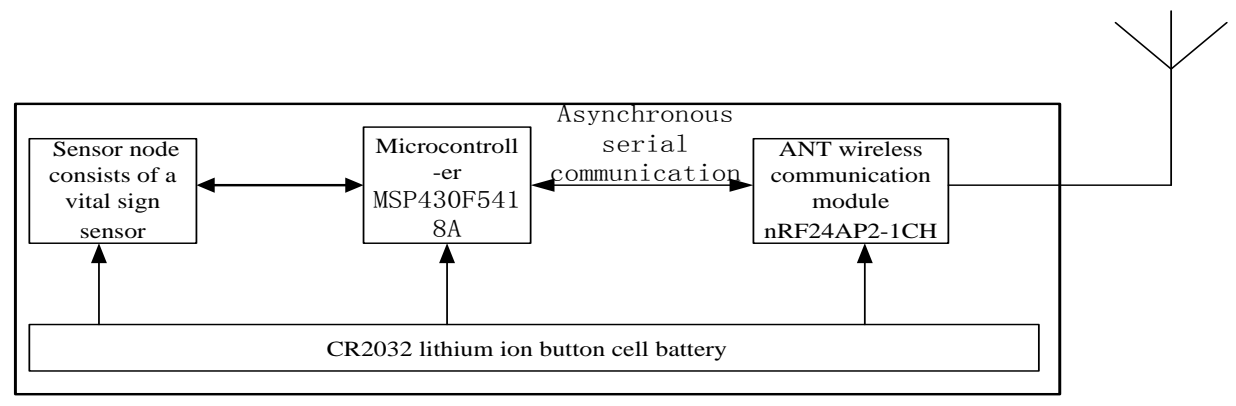

Figure 3. Structure Diagram of Sensor Nodes

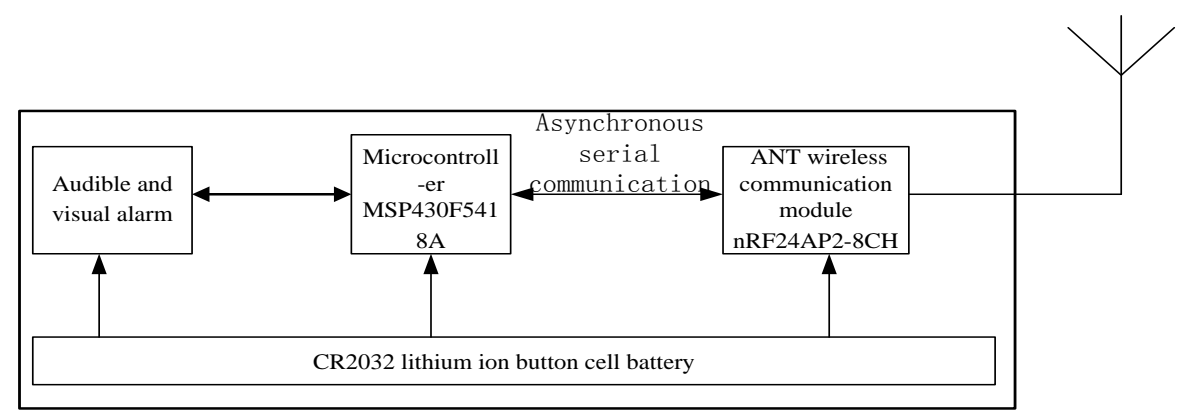

Figure 4. Structure Diagram of Central Node

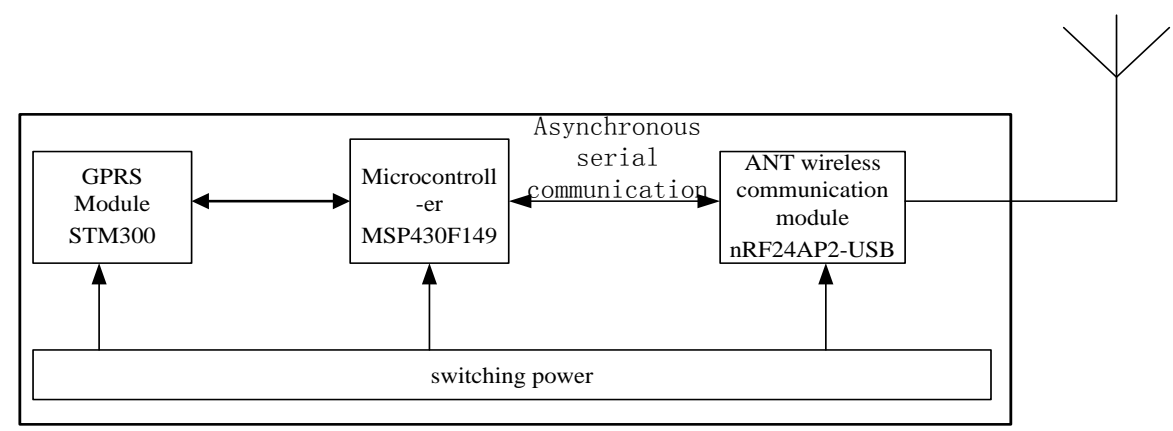

Figure 5. Structure Diagram of Routing Node

(2) Central Node: It consists of an audible and visual alarm, microcontroller MSP430F5418A, ANT wireless communication module nRF24AP2-8CH and CR2032 lithium ion button cell battery. nRF24AP2-8CH is an 8-channel ANT wireless network chip. In each ANT star-form network, the connection between the central node and sensor nodes allows at most 7 independent channels. Figure 4 is a structure diagram of the central node.

(3) Routing Node: It consists of microcontroller MSP430F149, ANT wireless communication module nRF24AP2-USB, GPRS Module STM300 and switching power supply. MSP430F149 features powerful processing capacity and calculation speed, low power consumption and rich external devices. GPRS module can be used for corresponding positioning of users. nRF24AP2-USB is ANT wireless network chip of USB interface 8 channels and can serve just as the routing node of ANT network. Figure 5 is the structure diagram of the routing node. 


\subsection{Network Design of the System}

\subsubsection{Topological Structure of the Network}

In order to build a wireless network featuring high reliability, good expansion performance and flexibility and reduce the complexity and cost of the network, a hybrid topological structure is adopted for the system. As the sensor nodes are close to the central node, direct communication can be realized. Therefore, a simple star network structure is used for the sensor nodes and the central node. As the central node is far from the routing node and may exceed the communication distance supported by ANT protocol, it is necessary to adopt a multi-hop data forwarding mode. Therefore, a complex mesh network structure is adopted between the routing node and the central node.

\subsubsection{Setting of Network Channels}

To complete the establishment of an ANT channel, as for such parameters as network ID, RF frequency, channel ID and channel cycle, connected nodes much have the same value, with the channel configuration process as shown in Figure 6.

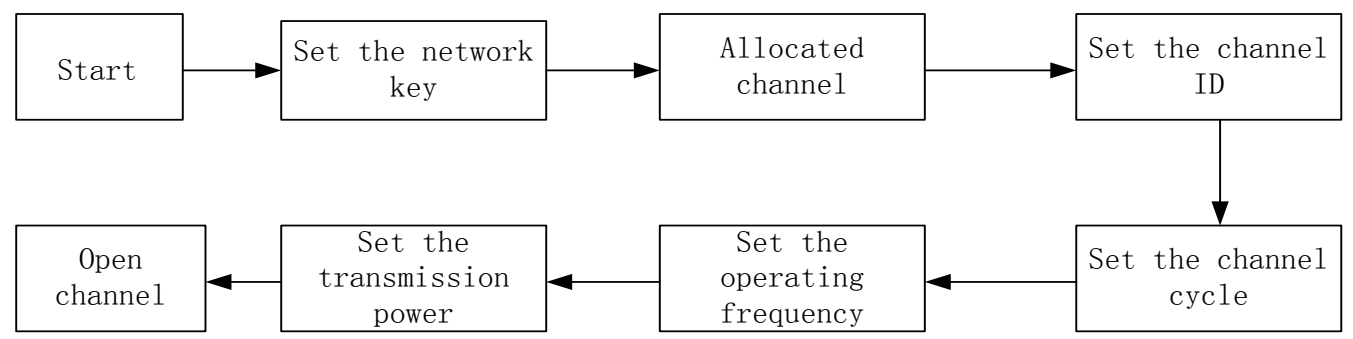

Figure 6. Channel Setting Process

Public network and public network key are adopted for the ANT network type, with setting value being 0 . Shared bi-directional channels are adopted for the channel type. As for the channel cycle, in order to reduce power consumption, the information transmission rate is set to be $0.5 \mathrm{~Hz}$. In order to ensure the network communication coverage scope, nRF24AP2 chip is adopted for the transmission power of the central node and the routing node, with the maximum transmission frequency being $20 \mathrm{dBm}$. As for the sensor nodes, in order to reduce power consumption, a transmission frequency of $10 \mathrm{dBm}$ is adopted. The working frequency of $2.4 \mathrm{GHz}$ is adopted and a broadcast data type is adopted.

\subsubsection{Node Configuration}

In the medical monitoring system, considering arranging one unique address for each node to make it easy to distinguish them and facilitate judgment of node distribution and type based on node address, field address field division is conducted and a certain meaning is endowed respectively. Each node is given a 2-byte address, with address allocation mode as shown in Table 2:

Table 2. Node Address Allocation

\begin{tabular}{|c|c|}
\hline User ID & Equipment ID \\
\hline$(12$ bit $)$ & $(4$ bit $)$ \\
\hline
\end{tabular}

"User ID" is used to distinguish among different users. For the same user, all nodes have the same user ID, 12 bits allocated to it. "Equipment ID" is used to distinguish among node types. For all nodes with the same function, they have the same equipment 
ID. For example, equipment ID 0 is used for the central node, and 1 for the sensor node used to collect body temperature and 2 for the sense node used to collect pulse. The address where user IDs and equipment IDs are all ones (i.e. node addresses are all ones) is reserved as the network access initial address, while the address where user IDs and equipment IDs are all zeros (i.e. node addresses are all zeros) is reserved for use during equipment address search.

\subsection{System Software Process}

\subsubsection{Sensor Node Software Design}

The main function of wireless sensor nodes is to send the data collected by sensors to the central node of the wireless network, with the sensor node software flow chart as shown in Figure 7.

\subsubsection{Central Node and Routing Node Software Design}

Central nodes are responsible for managing and processing the data sent by each sensor node and then sending them to routing nodes through ANT channel, and the routing nodes are responsible for managing and processing the data sent from each central node and then sending them to the data center terminal. Both have the same main process. The sensor node and routing (central) node software flow chart is as shown in Figure 8.

\subsubsection{Server Program Process}

The server in the medical center is responsible for storage, analysis, processing and dynamic display of physiological data sent by routing nodes, and the server compares the latest physiological parameter information with previous physiological data and normal physiological data. Based on data analysis and processing results, an order is given to the person being monitored to make corresponding adjustment. Additionally, the collected physiological data information tends to become inaccurate due to interference, so corresponding algorithm program is written to eliminate interference to obtain accurate data, with software structure block diagram as shown in Figure 9. 


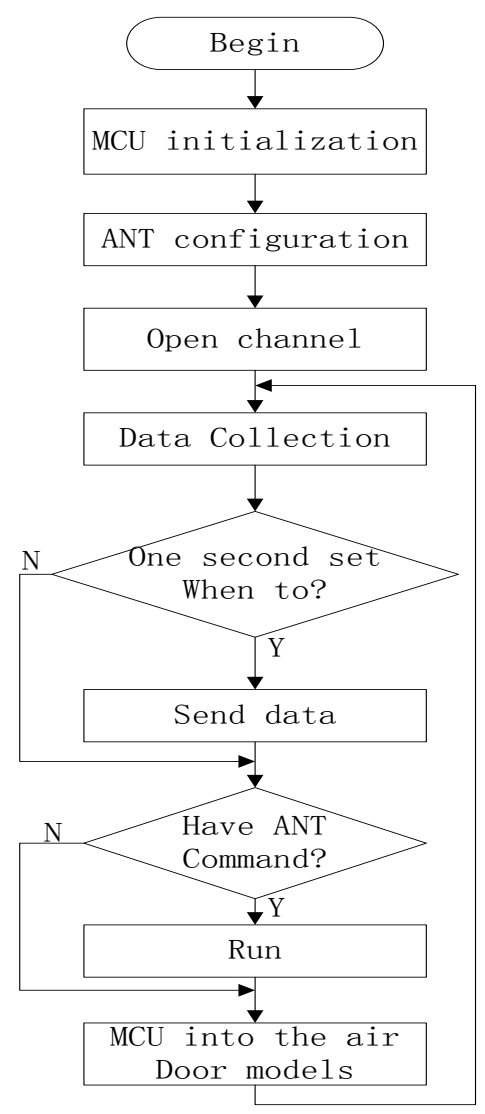

Figure7. Sensor Node Software Flow Chart

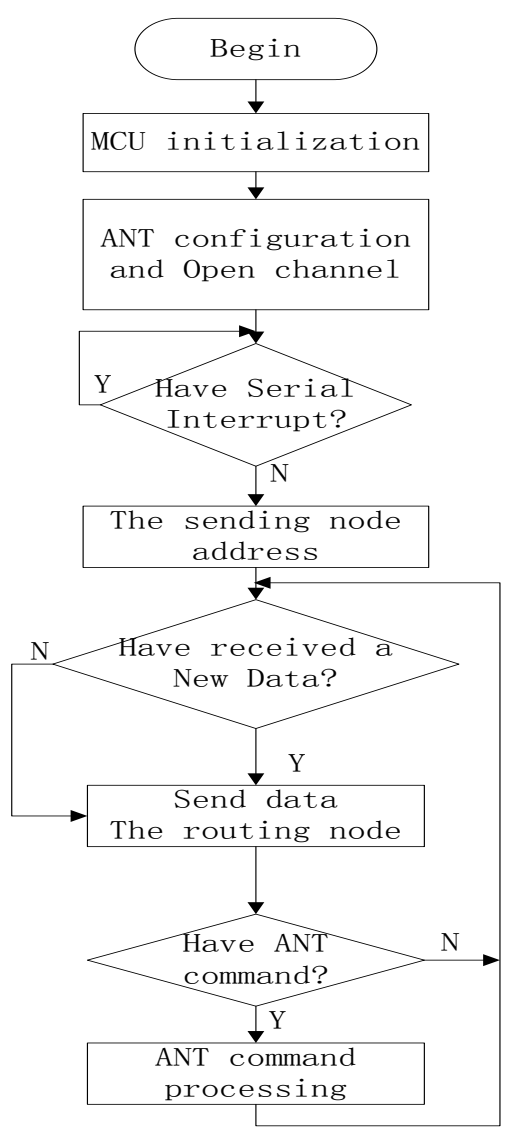

Figure8. Routing (Center) Node Software Flow Chart

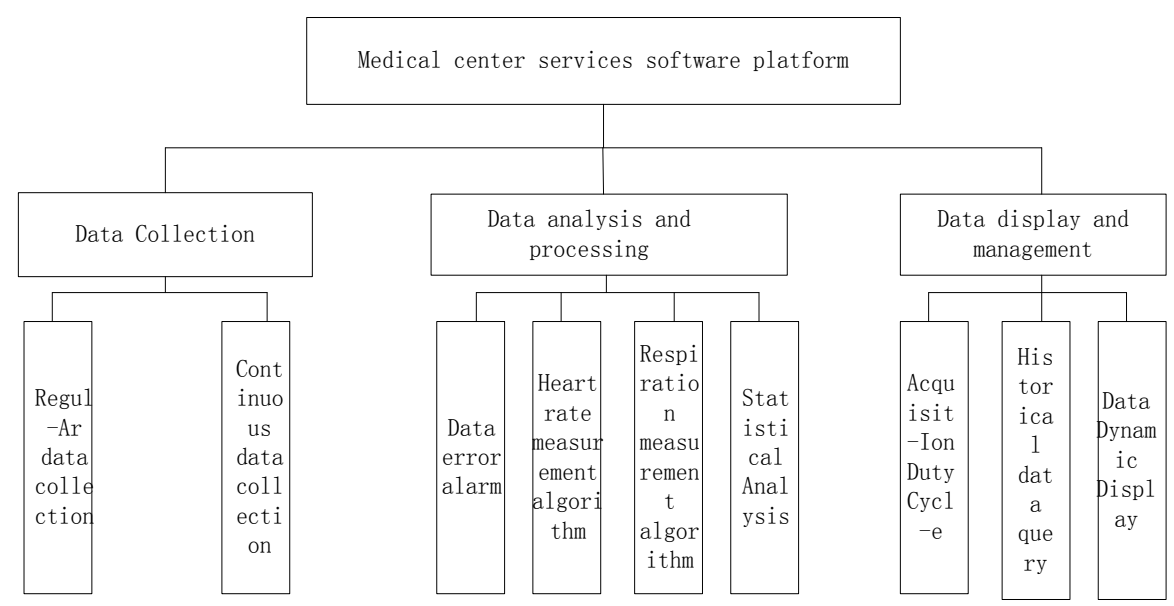

Figure 9. Server Software Structure Block Diagram

\section{ANT Network Performance Analysis}

\subsection{Reliability}

The reliability of network communication is determined based on the packet loss during data transmission. If the specified node fails to conduct data transmission according to ANT commands, it indicates packet loss during data transmission and no occurrence of the failure above indicates reliable data communication, with the test result 
as shown in Figure 10. The test result shows that ANT network has high communication reliability, which is why it is widely used in such fields as medical and health care fields.

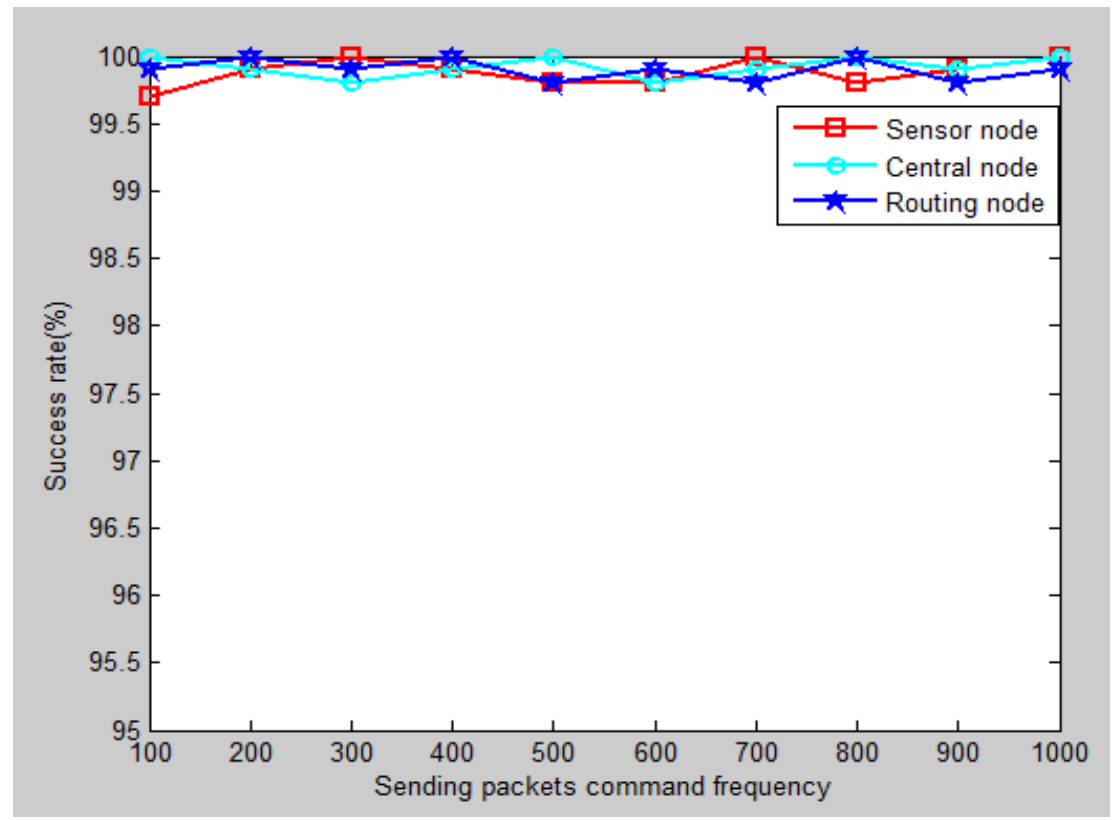

Figure 10. Network Reliability Test Result

\subsection{Power Consumption Estimation}

ANT nodes consume power mainly to maintain the work of SCM and ANT chips. The working current of MSP430F5418A is 330uA and low-power current is 2.1uA. The working current of MSP430F149 is $470 \mathrm{uA}$ and sleep power current is $0.1 \mathrm{uA}$. The working currents of different nRF24AP2 chips of sensor nodes, central nodes and routing nodes are $68 \mathrm{uA}, 103 \mathrm{uA}$ and $168 \mathrm{uA}$ respectively [11]. The average working current estimation of different nodes in ANT network is as shown in Table 3. The result indicates that ANT network has very low working power consumption.

\section{Table 4.1 Estimation of Average Working Current of Different Nodes in ANT Network}

\begin{tabular}{|c|c|}
\hline Node & Average current \\
\hline Sensor node & $39 \mathrm{uA}$ \\
\hline Central node & $48 \mathrm{uA}$ \\
\hline Routing node & $54 \mathrm{uA}$ \\
\hline
\end{tabular}

\section{Conclusion}

ANT protocol and related basic concepts are introduced in this paper, and at the same time the built-in wireless sending and receiving engine of nRF24AP2 is used as the communication module of ANT network, 430 series SCMs used as microprocessors for design of different network nodes and relevant software. Through testing of network system performance, corresponding functions of sensor nodes, central nodes and routing nodes at low power consumption, such as data acquisition, wireless networking and data transmission, can be realized. However, such problems as further reducing node volume 
and power consumption remain to be solved and continuous optimization can be conducted based on the existing design scheme.

\section{Acknowledgements}

This study was financed partially by Higher Education Key Laboratory for Measuring and Control Technology and Instrumentation of Heilongjiang Province, Harbin University of Science and Technology.

\section{References}

[1] T. Fei and Z. Yuanting, "Mobile Medical Care: Development Trend of Wearable Medical Instruments", Chinese Journal of Medical Instrumentation, vol. 5, no. 30, (2006), pp. 330-340.

[2] G. Yali and X. Jiqing, "Non-standard RF Protocol ANT", Journal of Chongqing Institute of Technology, vol. 3, no. 11, (2007).

[3] L. Li, Q. Zhu and Z. Shan, "Design ANT Low-power Wireless Networks", The Internet of Things Technologies, vol. 5, pp. 57-61, (2013).

[4] Y. Hao, M.K. Du, C.B. Li, "Improve TFRC Mechanism for Streaming Media in Wireless Network", Harbin Institute of Technology, vol. 2, (2011), pp. 40-43.

[5] T. Hui, "Principle and Advanced Applications of nRF24AP2 Single-chip ANT Ultra-low Power Wireless Networks", Beihang University Press, (2011).

[6] Y. Ming, G. Fei and G. Peng, "Design of nRF24AP2 Chip-based Wearable Medical Monitoring System", Electronics World, vol. 1, pp. 125-126, (2013).

[7] C. Yan-ming, W. Qiu-guang, "ZigBee Based Audio Transmission System", Harbin Institute of Technology, vol. 6, (2009), pp. 52-55.

[8] D. Fu, “ANT Protocol-based Wireless Sensor Network Research and Implementation”, HIT, (2012).

[9] Texas Instruments, MSP543xA, MSP430F541xA mixed signal microcontroller [EB/OL], (2013), http://www.ti.com.cn/cn/lit/ds/symlink/msp430f5418a.pdf.

[10] G. Dong, Q. Mingzhi, W. Weimin. "CC2430-based ZigBee Wireless Sensor Network Design and Implementation", The Internet of Things Technologies, vol. 3, no. 1, (2011), pp. 41-43.

[11] Dynastream Innovations Inc. ANT power estimator [EB/OL],
[ http://www.thisisant.com/developer/components/another-component/\#tab_power_estimator_tab.

(2013), 